\title{
IMAGING OF MAGELLANIC CLOUD PLANETARY NEBULAE WITH THE HUBBLE SPACE TELESCOPE
}

\author{
M. A. DOPITA ${ }^{1}$, S. J. MEATHERINGHAM ${ }^{1}$, P. R. WOOD ${ }^{1}$ \\ H. C. FORD ${ }^{2}$, R. C. BOHLIN ${ }^{2}$ \\ T. P. STECHER ${ }^{3}$, S. MARAN ${ }^{3}$ and J. P. HARRINGTON ${ }^{4}$ \\ ${ }^{1}$ Mt. Stromlo and Siding Springs Observatories, Institute of Advanced Studies, \\ The Australian National University, ${ }^{2}$ The Space Telescope Science Institute, \\ ${ }^{3}$ NASA Goddard Space Flight Center, ${ }^{4}$ The University of Maryland.
}

We have obtained Hubble Space Telescope (HST) Planetary Camera (PC) images of a number of Magellanic Cloud planetary nebulae. The objects, except for SMP 83 were observed as part of the Cycle I GO program. The observations were made in the [O III] $\lambda 5007 \AA$ line. The object SMP 83 , was observed as part of the GTO program, and in this case observations were also made in the $\mathrm{H} \alpha$ line using the F650N filter. In order to characterise the point spread function, a star was placed at the same point on the chip as the PN. This allowed us to determine the diameters of barely resolved PN in an accurate manner, by convolving the PSF with a function until it matched the appearance of the PN image. The results are given in Table 1.

Table 1: Dimensions and Dynamical Ages of the LMC PN inferred from HST Images.

\begin{tabular}{lccc}
\hline \hline Object & $\begin{array}{c}\text { Size } \\
\text { arc sec. })\end{array}$ & $\begin{array}{c}\text { Radius } \\
(\mathrm{cm})\end{array}$ & $\begin{array}{c}\text { Dynamical } \\
\text { Age }(\mathrm{yr})\end{array}$ \\
\hline SMP 02 & $0.25 \times 0.25$ & $9.32 \mathrm{e}+16$ & 2980 \\
SMP 08 & $0.06 \times 0.07$ & $2.43 \mathrm{e}+16$ & 310 \\
SMP 20 & $0.21 \times 0.43$ & $1.61 \mathrm{e}+17$ & 1969 \\
SMP 35 & $0.64 \times 0.86$ & $2.80 \mathrm{e}+17$ & 2150 \\
SMP 47 & $0.13 \times 0.21$ & $7.84 \mathrm{e}+16$ & 320 \\
SMP 72 & $1.72 \times 2.02$ & $6.97 \mathrm{e}+17$ & - \\
SMP 76 & $0.13 \times 0.13$ & $5.24 \mathrm{e}+16$ & 570 \\
SMP 83 & $0.70 \times 1.46$ & $4.04 \mathrm{e}+17$ & 1544 \\
SMP 85 & $0.07 \times 0.07$ & $2.60 \mathrm{e}+16$ & 1070 \\
SMP 87 & $0.79 \times 0.82$ & $3.02 \mathrm{e}+17$ & 2560 \\
SMP 96 & $0.17 \times 0.52$ & $1.94 \mathrm{e}+17$ & 1007 \\
\hline \hline
\end{tabular}

All the low excitation PN (E.C < 4) are compact objects, barely resolved with HST. These have [O III] diameters which are much smaller than was expected on the basis of photoionisation models, even when the fact that the $\mathrm{O}^{++}$zone occupies only a fraction of the ionised volume has been taken into account. This result can therefore be taken to mean that the $\mathrm{O}^{++}$zone is denser than the model.A second result is that the dynamical ages of these PN, as shown in Table 1, are much shorter than the evolutionary timescale of the central stars. This situation can only be maintained if there exists a dense, slowly expanding core of un-ionised gas in these PN, perhaps associated with shell ejection in the last helium flash. This gas would have to have an expansion velocity of only $\sim 1-3$ $\mathrm{km} . \mathrm{s}^{-1}$ in order to have remained compact to the present day.

The results described in this paper are based on observations with the NASA/ESA Hubble Space Telescope, obtained at the Space Telescope Science Institute, which is operated by the Association of Universities for Research in Astronomy, Inc., under NASA contract NAS5-26555. 